\title{
Plasmon-Enhanced Enzymatic Reactions: A Study of Nanoparticle-Enzyme Distance- and Nanoparticle Loading-Dependent Enzymatic Activity
}

\author{
Biebele Abel, Alice Akinsule, Canisha Andrews and Kadir Aslan* \\ Morgan State University, Department of Chemistry, 1700 East Cold Spring Lane, Baltimore, MD 21251 USA
}

* Corresponding author: Kadir.Aslan@morgan.edu(Kadir Aslan)

\begin{abstract}
A detailed investigation of the dependence of the efficiency of plasmon-enhanced enzymatic reactions on the distance between silver island films (SIFs) and horse radish peroxidase (HRP) enzyme and on the loading of SIFs on glass surfaces is presented. Three different extent of loading of SIFs on glass slides were used: 1) low, 2) medium and 3) high, which was characterized by using optical absorption spectroscopy and scanning electron microscopy. Streptavidin-linked HRP enzyme was deposited onto SIFs and glass slides by using three different strategies: strategy 1: biotin-avidin protein assay (distance between SIFs and HRP $=4-8 \mathrm{~nm}$ ), strategy 2: self assembled monolayers (SAMs) $(1-5 \mathrm{~nm})$, strategy 3: polymer layer $(1-5$ $\mathrm{nm}$ ). The efficiency of enzymatic conversion of O-phenylenediamine dihydrochloride (OPD) to a colored product by HRP on SIFs and glass surfaces was assessed by optical absorption spectroscopy. The distance between SIFs and HRP and the extent of loading of SIFs on the glass surfaces were shown to have significant effect on the efficiency of plasmon-enhanced enzymatic reactions. In this regard, up to an $\% 250$ increase in enzymatic conversion of OPD was observed from SIFs with high loading using strategy 1. In addition, we have studied the potential of repeated use of SIFs in plasmon-enhanced enzymatic reactions.
\end{abstract}

Keywords: Plasmonics, Silver Island Films, Silver Nanoparticles, Horse Radish Peroxidase, Metal-Enhanced Fluorescence.

Citation: B. Abel, et al. Plasmon-Enhanced Enzymatic Reactions: A Study of Nanoparticle-Enzyme Distance- and Nanoparticle LoadingDependent Enzymatic Activity. Nano Biomed. Eng. 2011, 3(3), 184-191 DOI: 10.5101/nbe.v3i3.p184-191.

\section{Introduction}

Plasmonics, a new research field based on the utilization of the surface plasmons of metallic structures (nanoparticles and planar surfaces), has been in use in many applications of bionanotechnology today. Plasmonic (surface plasmon-supporting metallic) structures can manipulate and transport electromagnetic energy and afford for the realization of more efficient biological and electronic processes. Surface Plasmon Resonance (SPR), Surface Enhanced Raman Scattering (SERS) and Surface Plasmon Fluorescence Spectroscopy (SPFS) are the most commonly known plasmonics technologies emerged in the last two decades. In addition, plasmonic nanoparticles have attracted the attention of scientists around the world due to their size (in the order of biomolecules themselves), amenability to the attachment of biological materials and their exceptional optical and electronic properties.
By combining the biological materials with plasmonic structures, one can create hybrid systems, which display biological and electronic functions at the same time. Moreover, these hybrid systems can enhance one or both of the functions of its components; for example, it was previously shown that plasmonic nanoparticles enhance the electro-catalytic response of a glucose oxidase system (glucose biosensor)[1] and the detection of various analytes of interest, such as hydrogen peroxide and uric acid [2], thiocholine [3], carcinoembryonic antigen [4] and DNA [5]. Several strategies for the immobilization of enzyme on plasmonic structures have been developed: layer-by-layer assembly[6], self-assembled monolayers[7], protein-antigen interactions[8], covalent attachment[9] and sol-gels[10]. Despite the existence of wide range of studies on enzyme/nanoparticle hybrids, the comparison of enzyme immobilization strategies and the 
effect of nanoparticle loading on surfaces on enzymatic activity were never reported.

In this paper, we present a detailed investigation of the dependence of enzymatic activity on the nanoparticle-enzyme distance and nanoparticle loading on planar surfaces. In this regard, three different SIFs were prepared (low, medium and high loading) on 3-Aminopropyltriethoxysilane (APTS)-coated glass slides. The loading of SIFs on glass slides were monitored by the absorbance of surface plasmon resonance peak of silver. These silvered surfaces and unsilvered (blank) APTS-coated glass slides (control experiment) were used for the comparison of three different enzyme immobilization strategies for plasmonenhanced enzymatic activity. A biotin-streptavidin protein assay (strategy 1), SAMs (strategy 2) and poly-1-lysine layer (strategy 3) were used to vary the distance of the enzyme from the silver surface. The enzymatic activity was followed by the colorimetric measurement of the product produced as a result of enzymatic conversion of o-phenylenediamine (OPD) on silvered surfaces and blank glass slides. It was found that up to an $\% 250$ increase in enzymatic conversion of OPD was observed from SIFs with high loading using strategy 1, providing a direct evidence that plasmon-enhanced enzymatic activity is highly dependent on the enzyme-nanoparticle distance and the extent of loading of SIFs. These findings will help the scientific community to better design enzyme-nanoparticle hybrid systems for applications in bionanotechnology.

\section{Materials}

Hexamethylenediamine $(98 \%), \mathrm{N}-(3-$ dimethylaminopropyl)-N'-ethylcarbodiimide hydrochloride (EDC), hydrogen peroxide (30 wt. \% solution in water), N-hydroxysulfosuccinimide sodium salt (NHS), poly-l-lysine solution $(0.1 \% \mathrm{w} / \mathrm{v}$ in water), sodium hydroxide $(99.99 \%)$, streptavidin-peroxidase from streptamyces avidinii (HRP-streptavidin), albuminbiotin-labeled-bovine (biotinylated-Bovine Serum Albumin) (b-BSA), bovine serum albumin (99\%) (BSA), D-glucose, 3-Aminopropyltriethoxysilane (APTS) (99\%), ammonium hydroxide (30\%) and nitric acid (A.C.S reagent grade) were all obtained from Sigma-Aldrich. Boric acid, borax (sodium borate), sodium phosphate dibasic and monobasic anhydrous were all obtained from Fisher Scientific. Culture well chambered cover glass (2 wells, $15 \mathrm{~mm}$ diameter, $2.0 \mathrm{~mm}$ deep) were obtained from Electron Microscopy Sciences (Hatfield, PA). Glass slides (micro slides, thickness: 0.96 to $1.06 \mathrm{~mm}$ ) was obtained from Corning Inc. O-phenylenediamine dihydrochloride (OPD) was obtained from Thermo-Scientific and was stored at $-20^{\circ} \mathrm{C}$ before use. Silver nitrate (A.C.S reagent grade) was purchased from Spectrum Chemical Mfg Corporation. Citric acid (analytical reagent grade) was purchased from Mallinckrodt Chemical Works. Sulfuric acid (A.C.S reagent grade) was purchased from Pharmco product Inc. Ethyl alcohol (200 proof) was purchased from PHARMCO. All chemicals were used as received. All solutions were prepared using deionized water obtained from Millipore Direct Q3 system.

\section{Methods}

(1) Silanization of Glass Slides using 3-aminopropyltriethoxysilane (APTS). The silanization procedure was done in accordance with the literature.[11],[12] All glass slides used in this work were cleaned using piranha solution (CAUTION: Sulfuric acid and Hydrogen peroxide in a ratio of 7:3 should be handled with care), the slides were later rinsed using deionized water and kept to dry at room temperature. The silanization solution was prepared using 5\% (w/v) APTS in 200 proof ethanol solution. The glass slides are then placed in a capped vial container that contains the prepared APTS solution for an hour, before rinsing with ethanol and water. The APTScoated slides were later kept to dry at room temperature.

(2) The Formation of Silver Island Films on APTScoated Glass Slides (Low, medium and high loading). SIFs were prepared according to the previously described procedure.[13] A solution of silver nitrate $(0.5 \mathrm{~g}$ in 60 $\mathrm{mL}$ of deionized water) was placed in a clean $100 \mathrm{~mL}$ glass beaker, which contains a Teflon-coated stir bar. The prepared solution is placed on a Corning stirring/ hot plate. While stirring 8 drops of freshly prepared $5 \%(\mathrm{w} / \mathrm{v})$ sodium hydroxide solution is added to the solution. A dark brown precipitates of silver particles is formed immediately. Ammonium hydroxide $(\sim 2 \mathrm{~mL})$ is then added, drop by drop, to redissolve the dark brown precipitates. The solution, which is clear, is then cooled to $5{ }^{\circ} \mathrm{C}$ by placing the beaker in an ice bath, followed by soaking the APTS-coated glass slides in the solution. A freshly prepared solution of D-glucose $(0.72 \mathrm{~g}$ in 15 $\mathrm{mL}$ of deionized water) is then added to the solution containing the APTS-coated glass slides. The temperature of the mixture is then warmed to $30^{\circ} \mathrm{C}$. The color of the mixture was observed to turn from yellow-green to yellow-brown, and the color of the slides becomes green, the SIFs-deposited APTS-coated glass slides were removed from the mixture, rinsed with deionized water, and dried using air. The different SIFs-deposition was done under different time intervals before removal, [low deposition ( $\sim 50$ seconds), medium deposition ( $\sim 2 \mathrm{~min})$, high deposition ( $\sim 4$ mins $)$ ].

(3) Preparation of Protein Assay (Biotin-Avidin) on SIFs-deposited APTS-coated glass slides and on Glass (Strategy 1). Biotin groups was introduced onto the glass and silver surfaces through biotinylated BSA, which results in the formation of a monolayer on the surface of glass and SIFs.[14] The schematic depiction of Strategy 1 is shown in Scheme 1. Culture well chamber cover glass ( 2 wells, $15 \mathrm{~mm}$ diameter, and $2.0 \mathrm{~mm}$ deep) was used to cover the slides before the addition of biotinylated BSA in pH 7 buffer $(\sim 200 \mu \mathrm{L})$ that incubated for approximately 30 minutes. Chambers were washed using deionized water to remove the unbound materials and dried using air. Bovine Serum Albumin (BSA, $0.5 \mathrm{mg} / \mathrm{mL}$ ) solution 
was then incubated inside the chambers for 30 minutes to minimize nonspecific binding of HRP-streptavidin to SIFs surfaces. HRP-streptavidin stock solution $(1000 \mu \mathrm{g} /$ $\mathrm{mL}$ ) is prepared using the sodium phosphate (monobasic) buffer solution at $\mathrm{pH} \mathrm{7,} \mathrm{the} \mathrm{stock} \mathrm{solution} \mathrm{is} \mathrm{then} \mathrm{diluted}$ 1:10 to a final concentration of $100 \mu \mathrm{g} / \mathrm{mL}$. Two hundred microliters of HRP-streptavidin solution was subsequently added into the chamber containing the monolayer of biotinylated BSA-coated with SIFs-deposited APTScoated glass slides. The incubation was carried out for 30 minutes at room temperature. The chambers were later washed using deionized water, dried with air to remove the unbounded HRP-streptavidin prior to the addition of o-phenylenediamine dihydrochloride.

(4) Preparation of SAMs on SIFs-deposited APTScoated Glass Slides (Strategy 2). A stock solution of 100 $\mathrm{mM}$ of hexamethylene diamine (HMA) in a borate buffer at $\mathrm{pH} 11.5$ (2.38 $\mathrm{g}$ of boric acid, $1.27 \mathrm{~g}$ borax and 5\% sodium hydroxide using $500 \mathrm{~mL}$ deionized water). The SIFs-deposited APTS-coated glass slides were immersed in HMA solution for 30 minutes. [Note: Incubation longer than 30 minutes results in the removal of SIFs on the surface of the glass]. The slides were rinsed with water to remove the unbounded HMA, and dried using air. Scheme 2 shows the schematic depiction of Strategy 2. Culture well chamber cover glass ( 2 wells, $15 \mathrm{~mm}$ diameter, and $2.0 \mathrm{~mm}$ deep) is then used to cover the slides before the addition HRP-streptavidin. The HRPstreptavidin was activated using the mixture of $200 \mathrm{mM}$ of N-hydroxysulfosuccinimide sodium salt (NHS) and 50 $\mathrm{mM}$ of N-(3-dimethylaminopropyl)-N'- ethylcarbodiimide hydrochloride (EDC) in sodium phosphate dibasic buffer solution at $\mathrm{pH} 8$ for 30 minutes. Unbound HRPstreptavidin was removed by washing with deionized water, before the addition of o-phenylenediamine dihydrochloride.

(5) Preparation of Poly-L-lysine Monolayer on SIFs-deposited APTS-coated Glass Slides (Strategy 3). The preparation of a Poly-L-lysine monolayer to attach proteins onto alkanethiol-modified gold surfaces has been previously described in literature.[15] Proteins are attached by direct cross-linking with amino groups of poly-L-lysine. Scheme 3 shows the schematic depiction of Strategy 3. The culture wells were used to cover the SIFsdeposited APTS-coated glass slides before the addition of poly-L-lysine $(\sim 200 \mu \mathrm{L})$ as described previously, which is kept for 30 minutes at room temperature. The slides were rinsed with deionized water and dried using air to remove the unbounded poly-L-lysine. HRP-streptavidin is then added after activation with the mixture of $200 \mathrm{mM}$ of N-hydroxysulfosuccinimide sodium salt (NHS) and 50 $\mathrm{mM}$ of N-(3-dimethylaminopropyl)-N'-ethylcarbodiimide hydrochloride (EDC). The slides were rinsed with deionized water and dried using air, before the addition of o-phenylenediamine dihydrochloride.

(6) Preparation of o-phenylenediamine dihydrochloride (OPD). OPD was prepared according to the manufacturer's product information. $10 \mathrm{mg}$ of OPD was dissolved in $10 \mathrm{~mL}$ of phosphate-citrate buffer, $\mathrm{pH}$ 5.0 ( $25.7 \mathrm{~mL}$ of $0.2 \mathrm{M}$ dibasic sodium phosphate, 24.3 $\mathrm{mL}$ of $0.1 \mathrm{M}$ citric acid and $50 \mathrm{~mL}$ of deionized water). The $\mathrm{pH}$ of the solution was adjusted to 5.0 using $5 \%$ sodium hydroxide, $10 \mu \mathrm{L}$ of $30 \%$ hydrogen peroxide. In all experiments, OPD concentration was set to $5.52 \mathrm{mM}$. A 2.5 M solution of sulfuric acid was used in stopping the reaction after the oxidation product of o-phenylenediamine and horseradish peroxidase was obtained,[16],[17],[18]. The absorbance of OPD was observed at $495 \mathrm{~nm}$ in all experiment, which was in accordance with the literature value.[19]

\section{Instrumentation}

All absorption measurements were done using Cary 50 Bio UV-Visible spectrophotometer, obtained from Varian, Inc. Scanning Electron Microscope images of SIFs were taken at University of Maryland Dental School Core Imaging Facility. Real-color photographs of SIFs before and after use were taken by a 5 MP digital camera.

\section{Results and Discussion}

The deposition of SIFs onto APTS-coated glass surfaces was carried out using Tollen's reaction scheme, which affords one to vary the extent of silver nanostructures deposited onto the glass surface by varying the time of growth of silver nanostructures. In this regard, SIFs with three different extent of loading (low, medium and high) was prepared. To prepare the SIFs with low loading, the glass slides were removed from the reaction medium immediately after the yellow-brown color formation in the medium ( $\sim 50$ seconds), while SIFs with medium and high loading were removed after $\sim 2$ mins and $\sim 4$ mins, respectively. Figure 1-Left shows the absorption spectrum for all SIFs, where an increase in SPR peak of the SIFs is observed as the loading of SIFs on glass slides is increased: low loading (SPR peak $=0.465)$, medium loading (0.800) and high loading (1.535). The SPR peak of SIFs with high loading is blue-shifted as compared to the other two surfaces, which implies that the size of SIFs with high loading is smaller than the other two surfaces. Figure 1-Right shows the real-color photographs of SIFs taken before use in the subsequent experiments, which visually demonstrate the change in loading of SIFs on glass slides.

In order to verify the observations made by absorption spectra and real-color photography, SIFs were also characterized by SEM (Supporting Information, Figure S1). SEM images show that the size of the SIFs was 50-90 $\mathrm{nm}$ for low and medium loading of SIFs and $\sim 50 \mathrm{~nm}$ for SIFs with high loading. SEM images also show that SIFs were deposited in a homogeneous manner. The low and medium loading SIFs appear as individual nanoparticles, where the distance between SIFs was shorter in high loading surfaces as compared to the surfaces with low and medium loading. These observations, although not absolutely, afforded for the direct comparison of the effect of loading of silver nanostructures on the enzymatic 
activity. It is also important to note that the effect of size of silver nanostructures were not attempted in this study due to the lack of control over the size of SIFs that can be deposited using Tollen's reaction scheme, which yields a distribution of sizes as observed here.

Fig. 2 shows the summary of results for Strategy 1 (Biotin-Avidin assay, Fig. 2-Top-Left). This assay scheme places the enzyme 4-8 nm away from the silver surface. It should be noted that HRP enzyme is oriented away from the surface, affording for all HRP to be involved in enzymatic activity. To study the enzymatic activity on glass slides and SIFs, a solution of OPD (5.52 mM) was placed on these surfaces. The interaction of OPD and HRP-streptavidin resulted in the change of color of the solution from colorless to light brown. Colored solution was removed after $\sim 2$ min of incubation and then placed in a vial that contains $1 \mathrm{~mL}$ of $2.5 \mathrm{M}$ concentration of sulfuric acid (stopping reagent). The solution was further diluted using sulfuric acid to yield absorbance values $<1$. The absorbance spectrum of the colored solution was measured after two uses of the same surface and are shown in Figure 2-Bottom. A control experiment, where SIFs was omitted from the glass surface, was also run twice to determine the background absorbance levels of OPD after the interaction with HRP-streptavidin without silver nanoparticles (straight and dashed lines for 1st and 2nd use in Figure 2-Top-Right, respectively). The average absorbance value measured from the control experiment in the first use was 0.095 , which was significantly lower than those for SIFs [Low (0.195), Medium (0.250), and High (0.255)]. Subsequently, it can be stated that SIFs are responsible for the $\sim 250 \%$ increase in the OPD absorbance. It is important to note that the amount of HRP was similar on both surfaces, which was previously demonstrated in another study from our laboratory[20]. However, the absorbance of OPD was decreased after the second use of the surfaces (Figure 2-Bottom and Top-Right), which could be attributed to the loss of enzymatic activity and/or loss enzyme from the surface due to the detachment of SIFs and HRP. To investigate these hypotheses, the absorption spectrum and real-color photographs of SIFs after 1st and 2nd use were taken and shown in Fig. 3. The real-color photographs and the absorbance value at SPR peak of SIFs show that SIFs were significantly detached from the surface after the 2nd use, which confirms the loss of SIFs and HRP from the surfaces. The loss SIFs from the surface is attributed to the extensive washing steps carried out during the experiments.

It is important to comment on the nature of surface plasmons of plasmonic structures with respect to their effect on biological functions. Plasmonic (e.g. gold, silver) nanoparticles are known to interact with light, which induces collective oscillations of the metal's electrons (i.e., surface plasmons).[21] The electric fields around the plasmonic nanoparticles are significantly increased, which is dependent on the wavelength of the incident light. It was shown that plasmonic nanoparticles can also interact with each other over distances up to 2.5 times of their diameter.[21] When a chromophore is placed in close proximity to plasmonic nanoparticles, two observations can be made: 1) electric field effect and 2) coupling of excited states energies of chromophores to surface plasmons (Fig. 3).[22] In the electric field effect, chromophores in close proximity $(<10 \mathrm{~nm})$ to the plasmonic nanoparticles are exposed to the increased electric fields in between and around the plasmonic nanoparticles, effectively resulting in significant increases in their absorption cross section. This lends itself to a subsequent increase in the excitation and eventually in the fluorescence emission from the chromophores, while the lifetime remains unchanged. In the second mechanism, where the excited-state energies of chromophores are partially transferred to surface plasmons, two distinct observations can be made for fluorescent species in close proximity to plasmonic nanoparticles: 1) an increase in the fluorescence emission from the metalfluorophore unified system with the spectral properties of the chromophores maintained, and 2) a reduction in the fluorescence lifetime, giving rise to improvements in the photostability of the chromophores. More recently, plasmonic nanoparticles are also predicted to increase the efficiency of chemical energy production of a photosynthetic system[23]. It was predicted that an increase in electron transfer processes occurs between the metal and photosynthetic system[23]. The predicted increase in the efficiency of the photosynthetic system was attributed to 1) plasmon enhancement of photon fields inside the light-absorbing chlorophyll molecules and 2) the energy transfer from chlorophylls to metal NPs. The first effect is thought to lead to strong enhancement of light absorption by the chlorophylls, whereas the second effect is thought to account for the reduction in the quantum yield of the system [23]. It is also thought that plasmonic nanoparticles increase the efficiency of electron transfer processes in enzymatic reactions, similar to the photosynthetic system described above.

Fig. 4 shows the summary of results for Strategy 2 (SAM, Fig. 4-Top-Left). Since HMA has one primary $\mathrm{NH}_{2}$ groups at both ends of the molecule: while one of the $\mathrm{NH}_{2}$ groups chemisorbed onto SIFs, the other one is used in the covalent attachment of HRP-streptavidin to SIFs. The covalent attachment of HRP-streptavidin is carried out after the activation of $-\mathrm{COOH}$ groups of HRP and streptavidin with NHS and EDC (to NHS esters) followed by displacement of the NHS groups by amine groups. The enzymatic activity was studied using the identical conditions described for strategy 1 in the previous paragraph. Fig. 4 shows an absorbance spectrum (Fig.4-Bottom) and the absorbance value (Fig. 4-TopRight) of OPD at $495 \mathrm{~nm}$ after 1 st and 2 nd use. A control experiment, where SIFs and HMA were omitted from the glass surface, was also run to determine the background absorbance levels of OPD after the interaction with HRPstreptavidin without SIFs (straight and dashed lines for 
$\underline{\text { Strategy } 1}$

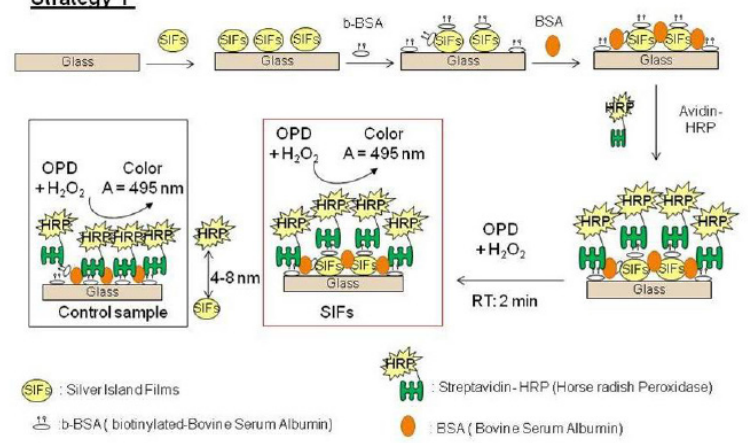

Scheme 1 Schematic depiction of Strategy 1: HRP enzyme is deposited on to SIFs using b-BSA-streptavidin interactions. Glass microscope slide is modified with 3-aminoproyltriethoxysilane, which contains terminal amine groups. In control samples SIFs are omitted from glass slide surfaces

$\underline{\text { Strategy } 3}$
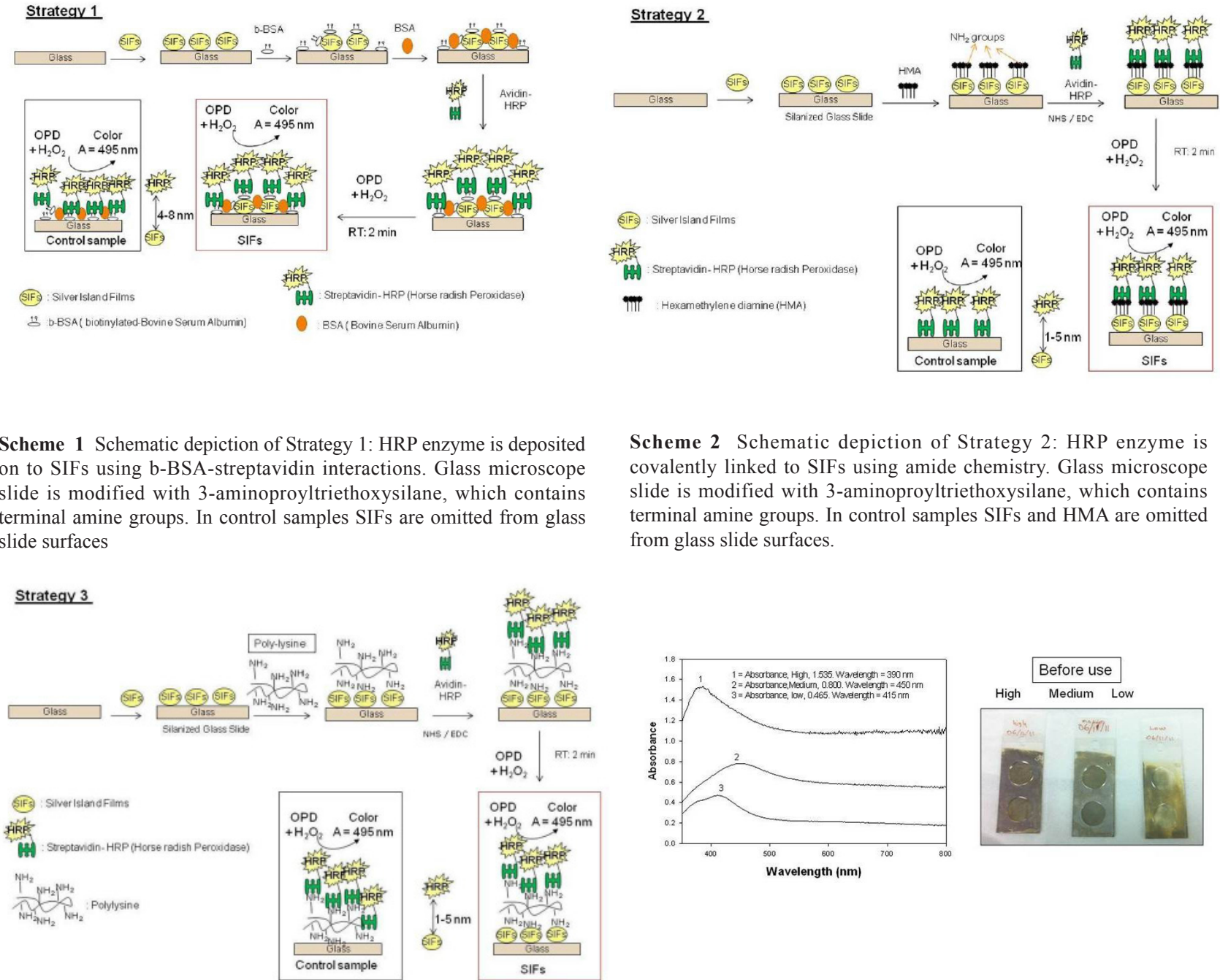

Scheme 2 Schematic depiction of Strategy 2: HRP enzyme is covalently linked to SIFs using amide chemistry. Glass microscope slide is modified with 3-aminoproyltriethoxysilane, which contains terminal amine groups. In control samples SIFs and HMA are omitted from glass slide surfaces.
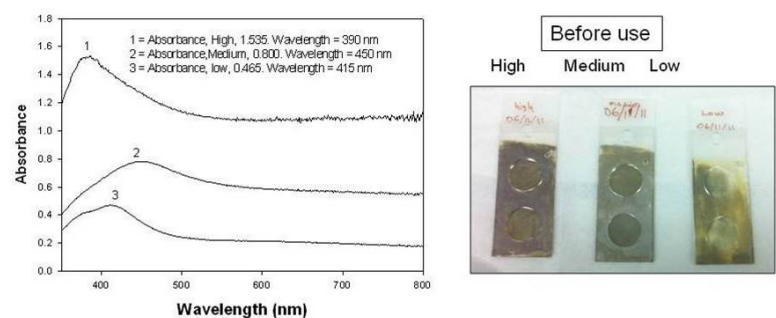

Scheme 3 Schematic depiction of Strategy 3: HRP enzyme is covalently linked to SIFs via poly-L-lysine groups. Glass microscope slide is modified with 3-aminoproyltriethoxysilane, which contains terminal amine groups. In control samples SIFs are omitted from glass slide surfaces.
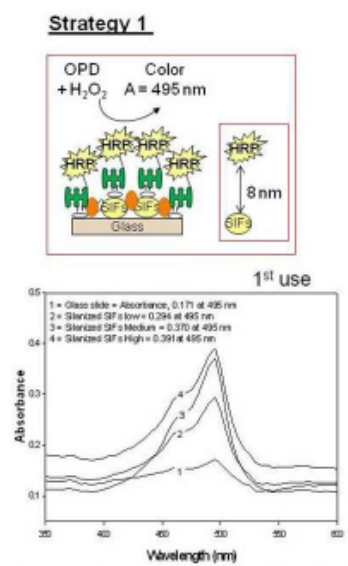
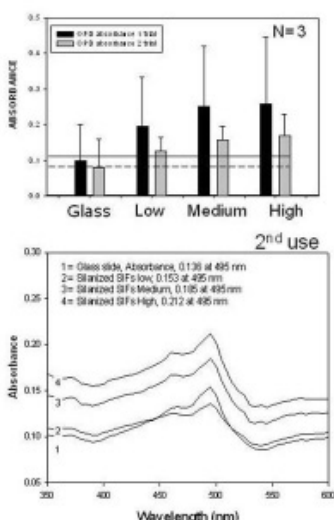

Fig. 2 Schematic depiction of Strategy 1 (Top-Left). Absorbance of OPD at $495 \mathrm{~nm}$ (Top-Right) and absorption spectrum (Bottom) of OPD on glass slide and SIFs (low, medium and high loading) after 1st and 2nd use used in Strategy 1.
Fig. 1 (Left) Absorption spectrum for SIFs: low, medium and high loading, (Right) Real-color photographs of SIFs: low, medium and high loading.

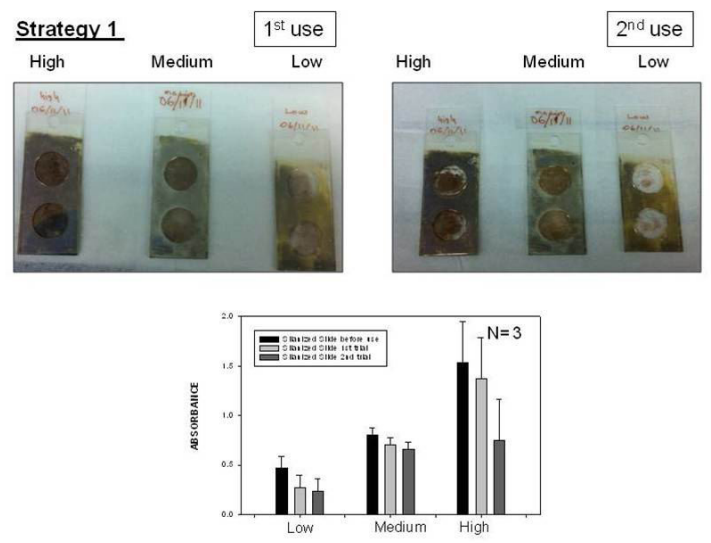

Fig. 3 Real-color photographs of SIFs used in Strategy 1: low, medium and high loading (Top-Left) before use, (Top-Middle) after 1st use, (Top-Right) after 2nd use. (Bottom) corresponding absorbance values of SIFs. 

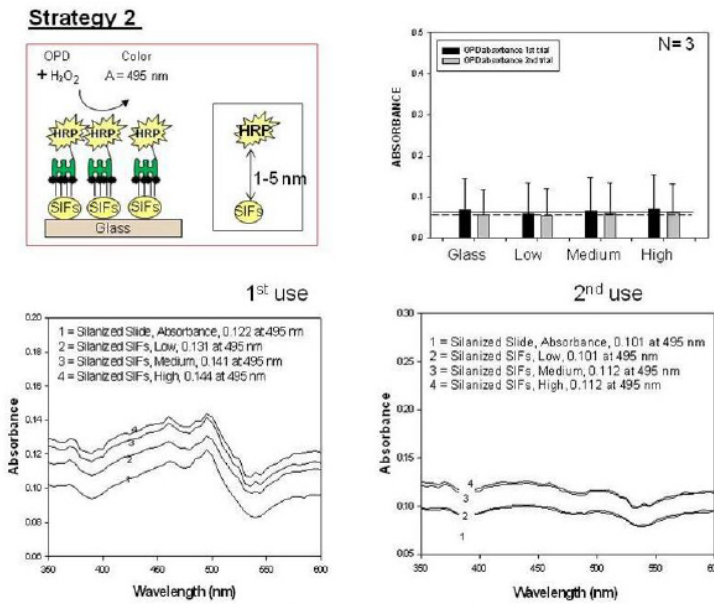

$2^{\text {nd }}$ use

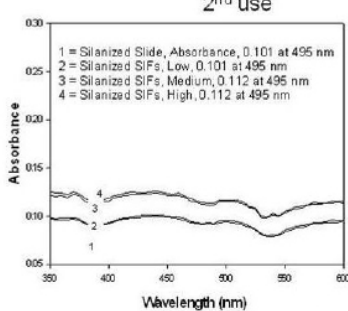

Fig. 4 Schematic depiction of Strategy 2 (Top-Left). Absorbance of OPD at $495 \mathrm{~nm}$ (Top-Right) and absorption spectrum (Bottom) of OPD on glass slide and SIFs (low, medium and high loading) after 1st and 2nd use used in Strategy 2.
Strategy 3

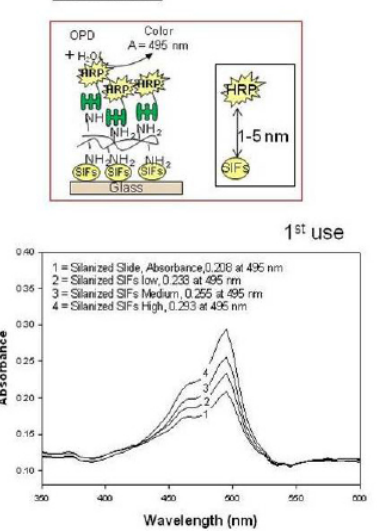

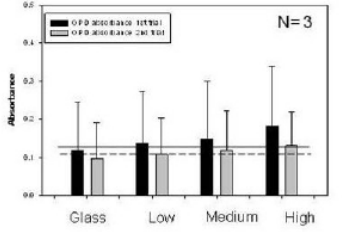

$2^{\text {nd }}$ use

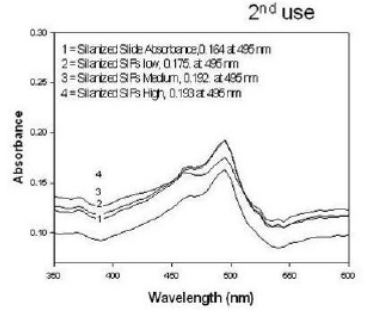

Fig. 6 Schematic depiction of Strategy 3 (Top-Left). Absorbance of OPD at $495 \mathrm{~nm}$ (Top-Right) and absorption spectrum (Bottom) of OPD on glass slide and SIFs (low, medium and high loading) after 1st and 2nd use used in Strategy 3.

1st and 2nd use in Figure 4-Top-Right, respectively). The absorbance value measured from the control experiment in the 1st use was 0.122 , which was slightly lower than those for SIFs. Moreover, absorbance values for OPD on SIFs and glass slides after 2nd use were similar to the background levels. Subsequently, it can be stated that strategy 2 did not yield significant enhancements of enzymatic activity. This may be attributed to the orientation of HRP to the surface: since this strategy employs covalent bonding between $\mathrm{NH}_{2}$ and $\mathrm{COOH}$ groups and HRP has also $\mathrm{COOH}$ groups, HRP is also expected to be involved in covalent bonding. One can expect that the covalent bonding of HRP to the surface results in the blockage of some of the enzyme's active sites, and thus, in the decrease in the number of HRP involving in enzymatic activity as compared to strategy 1. To investigate whether SIFs are detached from the surface as a result of washing and enzymatic activity in strategy 2 , the absorption spectrum and real-color photographs of SIFs after 1st and 2nd use were taken and shown in Figure 5. The real-color photographs and the absorbance value
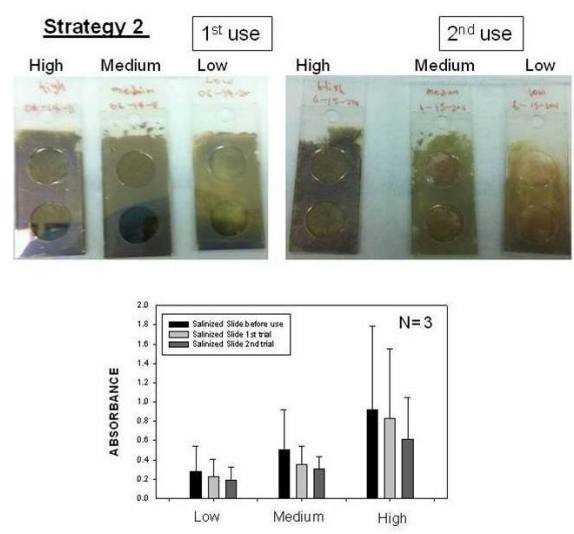

Fig. 5 Real-color photographs of SIFs used in Strategy 2: low, medium and high loading (Top-Left) before use, (Top-Middle) after 1st use, (Top-Right) after 2nd use. (Bottom) corresponding absorbance values of SIFs.
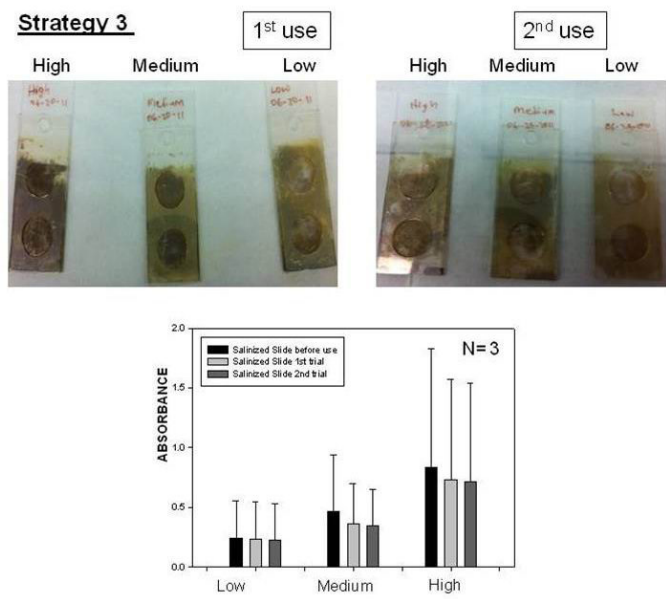

Fig. 7 Real-color photographs of SIFs used in Strategy 3: low, medium and high loading (Top-Left) before use, (Top-Middle) after 1st use, (Top-Right) after 2nd use. (Bottom) corresponding absorbance values of SIFs.

at SPR peak of SIFs show that SIFs were significantly detached from the surface after the 2nd use, which confirms the loss of SIFs and HRP from the surfaces. The loss SIFs from the surface is attributed to the extensive washing steps carried out during the experiments.

Figure 6 shows the summary of results for Strategy 3 (poly-L-lysine, Fig. 6-Top-Left). In this strategy, poly-Llysine is used to covalently attach HRP-streptavidin to the SIFs and glass surfaces. HRP-streptavidin was activated using NHS and EDC and was covalently linked to polyL-lysine using the same attachment method as described in strategy 2. Once again, both HRP and streptavidin molecules are thought to be involved in covalent attachment to SIFs, which results in blockage of the active sites of HRP. To study the enzymatic activity, OPD solution was added to the surfaces and the absorbance spectra of OPD after 1st and 2nd use were measured as shown in Figure 6-Bottom. A control experiment, where SIFs were omitted from the glass surface, was also run to determine the background absorbance levels of OPD after the interaction with HRP-streptavidin without 
silver nanoparticles (straight and dashed lines for 1st and 2nd use in Figure 6-Top-Right, respectively). A slight increase in OPD absorbance for SIFs as compared to glass surface was observed in both experiments, which indicates that the use of poly-L-lysine results in increase in the enzymatic activity of HRP. Since some of the HRP enzymes are involved in the covalent bonding to the surface, which results in the blockage of the enzyme's active sites, one would expect a decrease in the number of HRP involving in enzymatic activity.

To investigate whether SIFs are detached from the surface as a result of washing and enzymatic activity in strategy 3 , the absorption spectrum and real-color photographs of SIFs after 1st and 2nd use were taken and shown in Figure 7. The real-color photographs and the absorbance value at SPR peak of SIFs show that SIFs were slightly detached from the surface after two runs, which confirms that strategy 3 is the best immobilization technique in terms of the stability of SIFs among the techniques used in this study. However, it is important to remind that the largest increase in absorbance (i.e., enzymatic activity) was observed using strategy 1 . Work is currently underway to use these strategies in the development of nanoparticle-polymer-enzyme hybrid electrodes.

\section{Conclusions}

In this paper, we employed three different immobilization strategies for the investigation of the plasmon-enhanced enzymatic activity, which was assessed by optical absorption spectroscopic measurements of the interaction between OPD and HRP-streptavidin. The optical absorption spectroscopy studies revealed that the enzymatic activity was increased as the loading of SIFs on the glass surface was increased. Protein assay (strategy 1), which places the enzyme $\sim 4-8 \mathrm{~nm}$ away from the surface, was shown to be the most effective immobilization strategy in terms of obtaining the largest extent of plasmon-enhanced enzymatic activity. This was attributed to the use of plasmonic nanoparticles and the accessibility of the HRP active sites afforded by this immobilization technique. On the other hand, although a slight increase in enzymatic activity was observed in strategy 3 , it was concluded to be the best immobilization technique in terms of the stability of SIFs among the techniques used in this study, which shows that SIFs can be repeatedly used in plasmon-enhanced enzymatic reactions.

\section{Acknowledgements}

The project described was supported by Award Number 5-K25EB007565-05 from the National Institute of Biomedical Imaging and Bioengineering. The content is solely the responsibility of the authors and does not necessarily represent the official views of the National Institute of Biomedical Imaging and Bioengineering or the National Institutes of Health. The authors also acknowledge Department of Homeland Security, Office of University Programs, Science \& Technology Directorate: DHS 2008-ST-062-000011 for support.

\section{References}

1. Ren ML, Meng XW, Chen D, Tang FQ, Jiao J, Using silver nanoparticle to enhance current response of biosensor. Biosensors \& Bioelectronics. 2005; 21(3):433-437.http://dx.doi.org/10.1016/ j.bios.2004.08.052

2. Raj CR, Jena BK. Enzyme integrated silicate-Pt nanoparticle architecture: A versatile biosensing platform. Biosensors \& Bioelectronics. 2011; 26(6):2960-2966.http://dx.doi.org/10.1016/ j.bios. 2010.11 .046

3. Kirchhoff JR, Shulga O. An acetylcholinesterase enzyme electrode stabilized by an electrodeposited gold nanoparticle layer. Electrochem Commun. 2007, 9 (5), 935-940.http://dx.doi. org/10.1016/j.elecom.2006.11.021

4. Jia CP, Liu MY, Huang YY, Lou XH, Yao SH, Jin QH, Zhao JL, Xiang JQ. Highly sensitive protein detection using enzyme-labeled gold nanoparticle probes. Analyst. 2010; 135(2):327-331.http:// dx.doi.org/10.1039/b916629g

5. Nam JM, Jang KJ, Lee H, Jin HL, Park Y. Restriction-EnzymeCoded Gold-Nanoparticle Probes for Multiplexed DNA Detection. Small. 2009; 5(23):2665-2668.http://dx.doi.org/10.1002/ smll.200901105

6. Brett CMA, Crespilho FN, Ghica ME, Florescu M, Nart FC, Oliveira ON. A strategy for enzyme immobilization on layer-bylayer dendrimer-gold nanoparticle electrocatalytic membrane incorporating redox mediator. Electrochem Commun. 2006; 8(10): 1665-1670.http://dx.doi.org/10.1016/j.elecom.2006.07.032

7. Phadtare S, Shah S, Prabhune A, Wadgaonkar PP, Sastry M. Immobilization of Candida bombicola cells on free-standing organic-gold nanoparticle membranes and their use as enzyme sources in biotransformations. Biotechnol Progr. 2004; 20(6):18171824.http://dx.doi.org/10.1021/bp049792h

8. Addae SA, Pinard MA, Caglayan H, Cakmakyapan S, Caliskan D, Ozbay E, Aslan K. Rapid and Sensitive Colorimetric ELISA using Silver Nanoparticles, Microwaves and Split Ring Resonator Structures. Nano Biomed Eng 2010; 2(3):155-164.

9. Kim J, Kwon KY, Yang SB, Kong BS, Jung HT. High-performance biosensors based on enzyme precipitate coating in gold nanoparticleconjugated single-walled carbon nanotube network films. Carbon. 2010; 48(15):4504-4509.http://dx.doi.org/10.1016/j.carbon.2010. 08.027

10.Brennan JD, Luckham RE. Bioactive paper dipstick sensors for acetylcholinesterase inhibitors based on sol-gel/enzyme/gold nanoparticle composites. Analyst. 2010; 135(8):2028-2035. http://dx.doi.org/10.1039/c0an00283f

11. Horovitz O, Tomoaia G, Mocanu A, Yupsanis T, Tomoaia-Cotisel M. Protein binding to gold auto-assembled films. Gold Bull. 2007; 40 (4): 295-304.http://dx.doi.org/10.1007/BF03215603

12. Geddes CD, Previte MJR, Aslan K, Malyn SN. Microwave triggered metal enhanced chemiluminescence: Quantitative protein determination. Anal Chem 2006; 78(23):8020-8027.http://dx.doi.org/10. $1021 / \mathrm{ac} 061161+$

13. Aslan K, Geddes CD. Microwave-accelerated metal-enhanced fluorescence: Platform technology for ultrafast and ultrabright assays. Anal Chem 2005; 77(24):8057-8067.http://dx.doi. org/10.1021/ac0516077

14. Wilchek M, Bayer EA. The avidin-biotin complex in bioanalytical applications. Analytical Biochemistry. 1988; 171(1):1-32.http:// dx.doi.org/10.1016/0003-2697(88)90120-0

15.Jordan CE, Corn RM. Surface plasmon resonance imaging measurements of electrostatic biopolymer adsorption onto chemically modified gold surfaces. Anal Chem. 1997; 69(7):14491456.http://dx.doi.org/10.1021/ac961012z

16. Tarcha PJ, Chu VP, Whittern D. 2,3-Diaminophenazine is the product from the horseradish peroxidase-catalyzed oxidation of o-phenylenediamine. Anal Biochem. 1987; 165(1):230-3. http://dx.doi.org/10.1016/0003-2697(87)90224-7

17. Wolters G, Kuijpers L, Kacaki J, Schuurs A. Solid-phase enzymeimmunoassay for detection of hepatitis B surface antigen. J Clin Pathol 1976; 29(10):873-9.http://dx.doi.org/10.1136/jcp.29.10.873

18. Bovaird JH, Ngo TT, Lenhoff HM. Optimizing the o-phenylenediamine assay for horseradish peroxidase: effects of phosphate and $\mathrm{pH}$, substrate and enzyme concentrations, and stopping reagents. 
http://nanobe.org

Clin Chem 1982; 28(12):2423-6.

19. Bystryak SM, Mekler VM. Photochemical amplification for horseradish peroxidase-mediated immunosorbent assay. Anal Biochem 1992, 202 (2), 390-3.http://dx.doi.org/10.1016/00032697(92)90123-O

20. Grell TA, Ortiz EB, Das SR, Aslan K. Quantitative Comparison of Protein Surface Coverage on Glass Slides and Silver Island Films in Metal-Enhanced Fluorescence-based Biosensing Applications. Nano Biomedicine and Engineering. 2010; 2(3):165-170.

21. Kelly KL, Coronado E, Zhao LL, Schatz GC. The optical properties of metal nanoparticles: The influence of size, shape, and dielectric environment. Journal of Physical Chemistry B. 2003; 107(3):668677.http://dx.doi.org/10.1021/jp026731y
22. Aslan K, Previte MJ, Zhang Y, Geddes CD. Metal-Enhanced Fluorescence from Zinc Particulate Films. The Journal of Physical Chemistry C. 2008; 112(47):18368-18375.

23. Govorov AO, Carmeli I. Hybrid structures composed of photosynthetic system and metal nanoparticles: Plasmon enhancement effect. Nano Letters. 2007; 7(3):620-625.http://dx.doi.org/10.1021/ nl062528t

Copyright:(c) 2011 B. Abel, et al. This is an openaccess article distributed under the terms of the Creative Commons Attribution License, which permits unrestricted use, distribution, and reproduction in any medium, provided the original author and source are credited. 